\title{
Multivitamins and Nutritional Adequacy in Middle-Aged to Older Americans by Obesity Status
}

\section{Abstract}

Multivitamins are the most commonly consumed dietary supplement in the United States and worldwide. Micronutrient insufficiency and clinical deficiency are more common in middle-aged to older adults, and multivitamin use has been shown to improve status in this population. This analysis aimed to assess contributions of sporadic and consistent multivitamin use to total usual micronutrient intakes and associated nutritional biomarkers among middle-aged to older U.S. adults age ${ }^{3} 51$ years, stratified by obesity status. Self-reported dietary intake and laboratory measures from the National Health and Nutrition Examination Survey were used in these analyses. The National Cancer Institute method was used to assess usual intakes of 18 micronutrients. Compared with food alone, multivitamin use was associated with a lower prevalence of inadequacies and improved nutritional biomarker status for folate, iodine, selenium, and vitamins $\mathrm{B}_{6}, \mathrm{~B}_{12}$, and $\mathrm{D}$. Consistent use decreased the prevalence of inadequacy for most micronutrients assessed, except for those micronutrients typically not found (or in miniscule amounts) in standard multivitamin products. In addition to a lower prevalence of inadequacy for many micronutrients associated with consistent use of multivitamins, sporadic use decreased the prevalence of inadequacy for a greater number of micronutrients in obese versus nonobese individuals. Multivitamin use (sporadic and consistent) also increased the proportion of individuals who exceeded the tolerable upper intake level for folic acid to $8-10 \%$. Nutritional biomarker data indicate that obese individuals may be at greater risk of clinical deficiency in vitamins $\mathrm{B}_{6}$ and $\mathrm{D}$. Use of gender- and age-specific multivitamins may serve as a practical means to increase micronutrient status and decrease prevalences of clinical deficiency in the middle-aged to older population, particularly in those who are obese.

\section{Conflict of Interest}

C.L.F. and T.C.W. disclose unrestricted educational grant funding from Pfizer Consumer Healthcare for other research (not this specific research) within the last two-years. 